\section{Kaijer-Wilhelm-Bibel heilige Schrift}

Aiten und Teuen đeftaments

nat in indthe thringes

D. Martiu Eutbers.

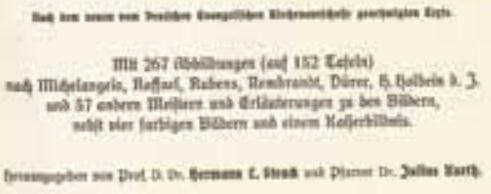

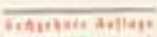

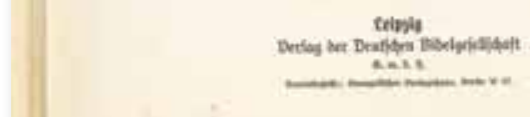

Abb. 11-12: Ba graph. 191701
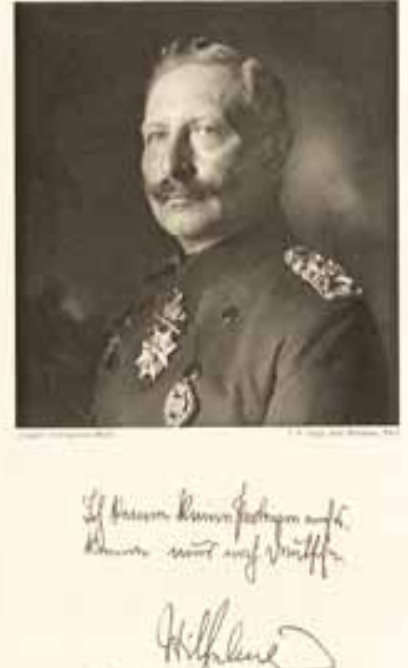

Yimblewe

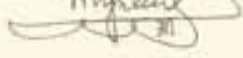

a66.

26/RENA

\title{
Digitalisierungsaktivitäten der Württembergischen Landesbibliothek - ein Zwischenstand
}

Nach Abschluss des Projekts "Vom Tresor in die Welt" konzentrieren sich die Digitalisierungsaktivitäten auf andere herausragende Bestandssegmente der historischen Sammlungen. Gegenwärtig (Stand: 25.2.2014) liegen 2.855 Dokumente mit 253.815 Seiten online vor. Der höchste prozentuale Anteil der Vorgänge entfällt auf die Graphiken (1.214 Titel online: $42,5 \%)$; bei den Seiten (1.220) verringert sich der Anteil entsprechend (0,5\%). Allerdings handelt es sich um Dokumente von jeweils nur einer Seite Umfang. Gemessen an der vorgesehenen Gesamtzahl von ca. 4.600 württembergischen Ortsansichten ist hier der Rest noch zu bearbeitender Titel am größten. Die Graphiken tragen erheblich zu dem hohen Anteil von Dokumenten mit Regionalbezug bei (ca. $41 \%$ ). Sie sind Teil der aus Mitteln des Lan- desdigitalisierungsprogramms finanzierten und für das Portal LEO-BW vorgesehenen Dokumente.

Umfangreich sowohl von der Zahl laufender Projekte als auch häufig von der Seitenzahl der Dokumente her sind die alten Drucke (792 Dokumente online: 27,7\%; 99.091 Seiten = 39\%). Darunter sind die knapp 400 digitalisierungsfähigen Inkunabeln mit unikalem Bestand in Deutschland sowie 162 VD16Drucke hervorzuheben. Im Rahmen des Landesdigitalisierungsprogramms wird ein der WLB Stuttgart zugeordnetes Segment an VD16-Drucken bearbeitet (473). Hinzu kommen etwa 200 Bibelausgaben aus der VD16-Nummernliste, von denen jedoch erst 69 online verfügbar sind. Insgesamt liegen 97 Bibeln mit 60.458 Seiten in digitaler Form vor $3,4 \%$ der 
Titel; 23,8\% der Seiten). Benutzeraufträge beziehen sich überproportional stark auf alte Drucke, von denen viele auf Württemberg bezogen sind.

Digitale Zweitformen sind zugänglich für 193 Handschriften mit 64.192 Seiten (6,8\% der Titel, 25,3\% der Seiten), darunter acht Musik-Handschriften. Hier sind besonders die illuminierten, meist mittelalterlichen Handschriften sowie Chorbücher und Stammbücher hervorzuheben. Auch bedeutende Exponate, die als Leihgaben an externe Ausstellungen gehen, werden digitalisiert. Die Umsetzung des DFG-Digitalisierungsprojekts „Bibelhandschriften im Folio-Format" muss noch begonnen werden. Um Handschriften handelt es sich zudem bei den Titeln aus dem Hölderlin-Archiv (368 Titel = 13,4\%; 10.050 Seiten $=4,0 \%)$. Motiviert auch durch das hundertjährige Jubiläum des Ersten Weltkriegs ist die verstärkte Digitalisierung seltener Drucke aus dem Bestand der Bibliothek für Zeitgeschichte (100 Titel $=3,1 \% ; 11.962$ Seiten $=4,7 \%$ ). Karten (31 Titel) und gedruckte Musikalien (41 Titel) stellen ein noch ausbaufähiges Segment dar.

Der Einstieg in die Massendigitalisierung ist durch die Digitalisierung und Erschließung der historischen württembergischen Landtagsprotokolle gelungen. Ein externer Dienstleister übernimmt nicht nur die Digitalisierung, sondern auch die Strukturdatenerschließung aufgrund von Vorgaben des Auftraggebers. Die bisher vorliegenden Seiten sind noch nicht freigeschaltet. Das Projekt wird aus Mitteln des Innovationsfonds „Kunst" sowie aus Bundesmitteln finanziert. Mit der Vergabe des Auftrags zur Digitalisierung der restlichen 131 Bände wird das Projekt im Verlauf des Jahres 2014 zum Abschluss kommen. Insgesamt handelt es sich um 1.229 bibliographische Einheiten zu den Jahrgängen 1797-1996 mit knapp 800.000 Seiten und ca. 31.000 Strukturdaten. Begleitet wird das digitale Angebot von historischen Hintergrundinformationen mit didaktischer Zielsetzung, die über die WLB-Homepage abrufbar sind.

Ein Dienstleister wird auch die historischen Stuttgarter Adressbücher digitalisieren und erschließen.
Dabei geht es um die Jahrgänge 1794 bis 1943 aus 18 bibliographischen Teilserien mit ca. 126.000 Seiten und über 16.000 Strukturdaten.

Im Haus hingegen werden ca. 66 Missionsbibeln aus der Bibelsammlung, d.h. eigens für die Verwendung in der Mission erstellte Bibelausgaben, in außereuropäischen Sprachen digitalisiert (ca. 24.300 Seiten, ca. 1.440 Strukturdaten). Häufig sind Missionsbibeln die ersten gedruckten Bücher überhaupt in einem bestimmten Territorium bzw. Sprachkreis. Begleitinformationen zur Geschichte der frühen Missionsbibeln (Stationen, Institutionen, Personen) sind bereits online verfügbar. ${ }^{1}$

Dokumentationsprojekte eigener Art sind die Arbeiten für das Wasserzeicheninformationssystem ${ }^{2}$ und die Einbanddatenbank (EBDB). ${ }^{3}$ Die Digitalisierung der zu beschreibenden Stücke (z.B. Durchreibungen von Werkzeugen) ist Voraussetzung für deren Nachweis und Zuordnung in der Datenbank. Der Nachweis eines Wasserzeichens wird jeweils verknüpft mit den in "Manuscripta Mediaevalia" enthaltenen Katalogisaten. Die WLB Stuttgart hat bisher 6.000 Wasserzeichen digitalisiert und dokumentiert. Die Einband-Durchreibungen von Ernst Kyriss sind für die gotischen Einbände bereits in der Einbanddatenbank enthalten. Dieses Angebot wird ab 2014 sukzessive ergänzt durch bisher nicht erfasstes Material zu Einbänden des 16. bis 17. Jahrhunderts (zunächst nur Bände aus der Hofbibliothek: 21 Mappen, ca. 2.000 Belegbände, ca. 1.700 Werkzeuge).

Zu den besonders wertvollen Beständen gehören auch die 156 großformatigen Bände der Sammlung Nicolai aus dem Bereich der Sammlung Karten / Graphik. Für 2014 ist die Digitalisierung und Erfassung eines Teilsegments davon (10 Bände) mit knapp 600 seltenen Karten geplant.

Umfangreiches Dokumentationsmaterial zum Ersten Weltkrieg ist von der Bibliothek für Zeitgeschichte für die Digitalisierung vorgesehen (Druckschriften, Bilder u.a.).

Christian Herrmann 